\title{
Correspondence
}

\section{Demodex folliculorum}

\section{To the Editorial Committee of the British Journal of Ophthalmology}

Sirs,-Attention is directed to a recent letter in your correspondence column by my friend and colleague from Denmark, Dr. M. Norn, in which he refers to his monograph on Demodex folliculorum, an eminently readable publication on this subject. Unfortunately it was not included in my bibliography as it was not to hand at the time of submitting my manuscript.

He makes particular reference to the caudate variant of Demodex folliculorum, an acarid which has lately been attracting a lot of attention. As mentioned in my article, the concept of a polymorphic presentation of the demodectic mite in man is not a recent one. Over a century ago Erasmus Wilson recorded this in the English literature while Simon of Berlin introduced it to German scientific circles.

Recent studies from the University of Massachusetts (Desch and Nutting, 1972) are of significance to the ophthalmologist interested in acarology. Desch and Nutting, in an exhaustive study of hundreds of sections, record that the two species of Demodex folliculorum occupy different sites in the pilosebaceous unit.

They note that the caudate variant, better known as Demodex folliculorum brevis, resides in the sebaceous gland, apparently in time digesting its contents. The larger more common parasite inhabits the follicle proper, producing undercutting of the epithelial cells. In heavy cases of infestation they produce distension of the follicle and metaplasia of the epithelial lining cells.

These observations bring to mind some unanswered questions pertaining to Demodox folliculorum. One concerns selective damage to the sebaceous gland and its effect on metabolism of hair. It is difficult to know what role lubrication of hair has in Homo sapiens, but those who have studied comparative anatomy at the Royal College of Surgeons will recall with alacrity the importance of this mechanism in lower species especially in felines.

Finally, it has yet to be seen if heavy infestation involving the meibomian glands has any effect on the maintenance of the lipid layer of tear film which is concerned with preventing evaporation.

In closing, it is stimulating to note that other research centres throughout the world are displaying interest in this commonly occurring parasite infesting man.

I 3 WICKHAM TERRACE

BRISBANE, QUEENSLAND 4000, AUSTRALIA

Fune 6, 1972

\section{Reference}

Desch, (.., and nuiting, w. B. (1972) .7. Parasit., 58, i69 